\section{Improving maintenance medication adherence in adult inflammatory bowel disease patients: a pilot study}

\author{
Michelle L. Matteson-Kome,1 Jessica \\ Winn,2 Matthew L. Bechtold, 1 Jack D. \\ Bragg, 1 Cynthia L. Russell ${ }^{3}$ \\ 1Division of Gastroenterology and \\ Hepatology, Sinclair School of Nursing, \\ University of Missouri, Columbia, MO; \\ 2Department of Internal Medicine, \\ University of Missouri, Columbia, MO; \\ 3School of Nursing, University of \\ Missouri-Kansas City, MO, USA
}

\section{Abstract}

Medication nonadherence in inflammatory bowel disease (IBD) may lead to suboptimal control of the disease, decreased quality of life, and poor outcomes. This pilot study evaluated the feasibility, intervention mechanism, and potential effectiveness of a three-month continuous self-improvement (CSI) intervention to enhance medication adherence (MA) in adult nonadherent IBD patients. Adult IBD patients taking a daily or twice-daily dosed maintenance medication were screened electronically for two months to determine baseline MA levels. Nonadherent IBD participants were randomized to the CSI or the attention control (AC) intervention and monitored for three months. The CSI intervention consisted of a data evaluation and system refinement process in which system changes were identified and implemented. The AC group was given educational information regarding IBD disease process, extra-intestinal manifestations of IBD, and medical therapy. Demographic statistics, change scores for within and betweengroup differences, and effect size estimates were calculated. Nine nonadherent participants (medication adherence score <0.85) were eligible for randomization. The intervention was found feasible and acceptable. Although no statistically significant improvement in MA was found $(\mathrm{P}=0.14)$, adherence improved in 3 of 4 of the CSI group and 1 of 2 in the attention control group. The effect size calculation of 1.9 will determine the sample size for future study. The results of this pilot study showed the intervention was feasible and had a positive effect on MA change score and adherence levels. A larger fully powered study is needed to test of the effectiveness of this innovative intervention.

\section{Introduction}

Adherence is defined as the extent to which a person's behavior - taking medication, following a diet, and/or executing lifestyle changes, corresponds with agreed recommendations from a health care provider. ${ }^{1}$ Medication adherence in chronic diseases averages 50\% in developed countries. ${ }^{1}$ Poor adherence in chronic disease results in increased health care costs and poor outcomes. ${ }^{1}$ Medication adherence interventions have had inconsistent results across chronic illnesses, and no patient-level intervention has consistently enhanced adherence.2-5 Inflammatory bowel disease (IBD), which includes Crohn's disease (CD), ulcerative colitis (UC), and indeterminate colitis, is characterized by periods of relapse and remission. In the United States, IBD is a chronic disease affecting approximately 1.4 million people, with Crohn's disease affecting approximately 26.0-198.5 individuals per 100,000 and UC affecting approximately 11 per $100,000.6,7$ The medication regimen for IBD is complex and lifelong which increases medication nonadherence rates. ${ }^{8}$ Current medication nonadherence rates in IBD range from 7 to $72 \% .{ }^{9}$ IBD medication nonadherence can lead to suboptimal control of the disease, decreased quality of life, and increased morbidity. ${ }^{10}$

Continuous self-improvement (CSI), an innovative intervention for affecting health behavior change, is based on systems theory. A recent systematic review of CSI intervention literature found that it helped change health behaviors, specifically life-style management (exercise and weight loss) and chronic diseases (asthma and kidney transplantation). ${ }^{11}$. CSI is a unique, systems-focused intervention that seeks to change behavior that focuses on patients' personal systems rather than on their motivation or intention. ${ }^{12,13}$ This study's purpose is to evaluate the feasibility, intervention mechanism, and potential effectiveness of the CSI intervention in adult nonadherent participants in a mid-western IBD clinic.

\section{Materials and Methods}

A pilot randomized controlled trial tested the feasibility, intervention mechanism, and possible effectiveness of a three month CSI intervention to improve medication adherence in adult nonadherent IBD patients. Participants were recruited from a mid-western outpatient IBD clinic by the primary investigator (PI) and/or the research assistant (RA). Initial inclusion criteria were: age 18 years or older; diagnosis of ulcerative colitis or Crohn's disease (diagnosed based on clinical/histology
Correspondence: Michelle L. Matteson-Kome, Division of Gastroenterology and Hepatology, University of Missouri, 101 Fairview, Columbia, M0 65203, USA.

Tel. +1.573.884.8181 - Fax: +1.573 .884 .8200 .

E-mail: mattesonml@health.missouri.edu

Key words: adherence, inflammatory bowel disease, intervention.

Acknowledgments: Sigma Theta Tau International and Sigma Theta Tau-Alpha Iota for grant funding received for this study. Dr. Jamal Ibdah and the staff at the Digestive Health Center, for support and assistance with this study.

Funding: Sigma Theta Tau International Small Grant; Sigma Theta Tau-Alpha Iota Chapter.

Contributions: MM, study design, collection of data, analysis of data, writing of the manuscript; JW, enrollment of patients, collection of data, manuscript review; $\mathrm{MB}$, analysis of data, manuscript preparation and review; JB, enrollment of patients, manuscript preparation and review; CR, study design, analysis of data, writing of manuscript and review.

Conflict of interests: the authors declare no potential conflict of interests.

Received for publication: 18 February 2013. Revision received: 11 March 2013.

Accepted for publication: 11 March 2013.

This work is licensed under a Creative Commons Attribution NonCommercial 3.0 License (CC BYNC 3.0).

(C) Copyright M.L. Matteson-Kome et al., 2014 Licensee PAGEPress, Italy

Health Psychology Research 2014; 2:1389 doi:10.4081/hpr.2014.1389

evidence); currently on IBD maintenance medication therapy (immunosuppressant or 5aminosalicylic acid); ability to speak, hear, and understand English; able to open Medication Event Monitoring System (MEMS) caps; and Mini-Mental Status Examination (MMSE) score greater than or equal to 24 . Of the 41 adult IBD patients who were approached to enter the study, 38 participants consented and enrolled, and three refused to participate due to time or schedule conflicts (Figure 1).

The independent variable was the CSI intervention. Personal system thinking is conceptually defined as the process of understanding how people and circumstances are linked. ${ }^{14}$ This approach attempts to improve patients' systems by creating and maintaining a behavior, such as taking medication. $12,13,15$ Through the data evaluation and system refinement process called Plan, Do, Check, Act (PDCA), patients' personal systems are identified and 
changes implemented. Further CSI theoretical detail is described elsewhere (Figure 2). ${ }^{11}$

The CSI process fosters ritualistic and habitual health behaviors and requires less effort, motivation, and intention to maintain the changes. For each participant, the PI performed a one-time face-to-face CSI intervention assessing the screening MEMS data after a brief personal system theory power-point presentation in the IBD clinic. The PI and participant analyzed electronic monitoring data for patterns of nonadherence, identifying potential personal system changes. System changes suggested by participants were performed to the best of their ability throughout the three-month study.

The control group received an attention control intervention. A one-time face-to-face educational session performed by the PI included an electronic slide presentation with a handout based on information from the Crohn's and Colitis Foundation of America (www.ccfa.org). IBD education topics included IBD medical therapy actions and side effects, extra-intestinal manifestations of IBD, and surgical modalities utilized in IBD.

\section{Dependent variables}

Feasibility was determined using a 15-question, open-ended, written survey completed by the participants. The survey was used in a previous pilot study. 12

The intervention mechanism was evaluated by the Systems Thinking Survey. This survey was designed to measure change in personal systems thinking, which is a foundational concept of the CSI intervention. This survey, which was originally developed to evaluate physical exercise systems thinking, was adapted for medication taking by two experts in the field (CR and MM). This 17-question survey employed a 5-point Likert scale (1=not important, $5=$ very important). Scores range from 17 to 85 with higher scores indicating higher lev- els of systems thinking. No psychometric data are available for the survey. 12

The medication nonadherence outcome was measured by the Medication Event Monitoring System (MEMS, MEMS Track Cap, Apres Corp., Union City, CA, USA) electronic bottle cap. The MEMS cap's effectiveness and reliability are well documented. ${ }^{16-20}$ The MEMS score utilizes binary data $(1=y e s ; 0=$ no) for evaluating dosing of medications. However, binary data cannot evaluate the timing of medication taking. In order to assess dosing and timing, a medication adherence score was determined to be superior for capturing the dynamics of medication taking. ${ }^{21}$

A window of time was used to determine medication adherence which then allowed calculation of a medication adherence score. The on-time window for twice daily dosing was calculated as $\pm 25 \%$ of the prescribed medication dosing interval; the early or late window was $\pm 50 \%$, and the missed window was a dose not taken within $\pm 50 \%$ of the prescribed time. This calculation approach, which captures the variability of adherence in timing and dosing, had been successfully used on twice-daily dosed medications. ${ }^{21}$ However, this approach did not allow calculation of adherence scores for medications dosed three or four times a day, because the dosing windows overlap. Consequently, 15 consented participants who took maintenance medication three or four times a day were withdrawn from the study (Figure 1). The Institutional Review Board was contacted and inclusion criteria were changed to reflect the new inclusion criteria of daily or twice-daily dosed maintenance medication therapy.

Participants received MEMS diaries to record accidental openings or purposeful opens when medications were not taken (such as removing a pill to be taken later or refilling the medication bottle). These data are used to correct the MEMS data prior to analysis which increases the internal validity of the MEMS instrument.

The study was approved by the Institutional Review Board at the University of Missouri. Informed consent was obtained from all study participants, and no study participants were harmed during the study. A two-month screening phase identified participants who were nonadherent to their maintenance therapy medications. MEMS caps were utilized on participants' physician-prescribed maintenance therapy medication; when more than one medication was eligible for monitoring, the twice daily medication was monitored. For participants with medication planners, a candy reminder was placed in the planner to remind them to take maintenance therapy from the MEMS bottle. Nonadherence was operationally defined as a medication adherence score less than 0.85 based on a sixty day screening period, a value that was empirically developed and utilized in previous studies. 16 The first 30 days of the screening period were removed to account for possible weak intervention effect of the MEMS cap.22 At the completion of the screening phase, adherent participants (medication adherence score $>0.85$ ) were thanked for their participation by the PI, praised for their adherence, given a $\$ 10$ gift card, and exited from the study. Those who were nonadherent were randomized.

The PI used sealed numbered envelopes to block randomize nonadherent participants in sequence of admission into the trial's CSI intervention or attention control group. Participants were blinded to group assignment. For the participants' convenience, after 3 months, a self-addressed stamped envelope was mailed to the participant to facilitate the return of the MEMS cap, MEMS diary, feasibility survey, and System Thinking Survey. After participants returned the MEMS cap, forms, and diary, and they were given a $\$ 10$ gift card for participating in the study.

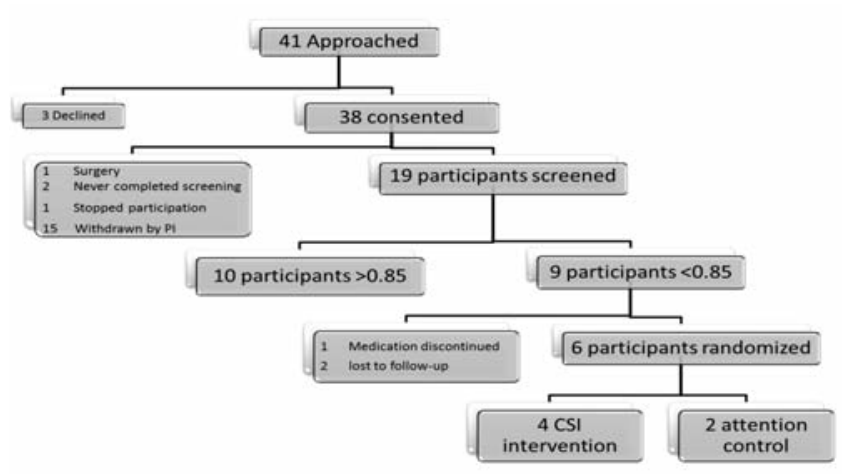

Figure 1. Patient flow diagram.

\section{Continuous Self-improvement}

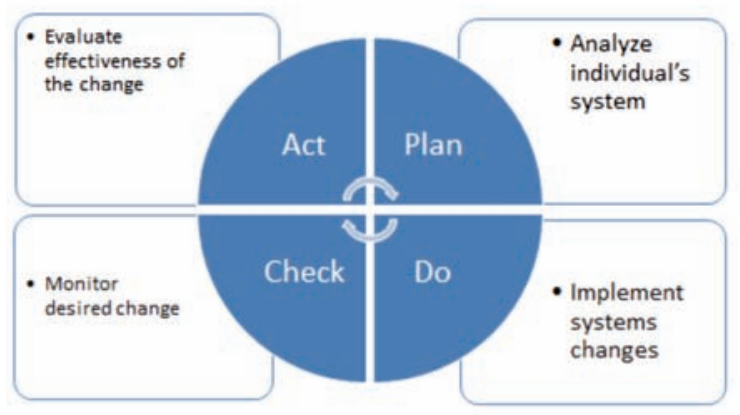

Figure 2. Plan, Do, Check, Act cycle. 
Data were cleaned and SAS v9.1 (SAS Institute, Inc., Cary, NC, USA) was used by the project biostatistician to conduct all data analyses. Descriptive statistics were performed to characterize the sample. Feasibility was evaluated by frequency of responses. Personal systems thinking was evaluated by systems survey mean and standard deviations of the change scores. Medication adherence change scores (changes between the two time points) as well as $95 \%$ confidence intervals for differences within and between the two groups were computed. Change scores within a group and between two groups were compared using the paired t-test. In keeping with the intention of a pilot study, we were interested in obtaining estimates for the effect size. From the means and standard deviations, the effect size was calculated. The estimate of the mean group difference as well as estimates of the standard deviation of the group differences in MEMS scores will be useful in determining appropriate sample size for a future large-scale study and for comparison to other studies.

\section{Results}

Participants were recruited from November 2010 to April 2011. Baseline demographic categorical data of the 19 participants is located in Table 1. IBD continuous demographic data are noted in Table 2. Participants' average age was 44.8 years ( $\mathrm{SD}=13.0$ years), with a range of 21 68 years. The age of IBD onset averaged 32.8 years $(\mathrm{SD}=12.7$ years $)$, with a range of $18-63$ years. The number of medications taken by participants averaged $5.2(\mathrm{SD}=4.9)$, with a range of 1 to 16 .

Of the 19 participants completing the screening period, nine $(47.4 \%)$ were found to be nonadherent. Screening MEMS scores across the 19 participants averaged 0.813 $(\mathrm{SD}=0.153)$, with a range of 0.475 to 0.987 . Three nonadherent participants (3/9) did not complete the study, as one was taken off her immunosuppressant and two were lost to follow-up despite multiple attempts by the PI to contact them via telephone and mail. Six of the nine participants were randomized, four participants to the CSI group and two to attention control. The duration of the one-time face-toface intervention ranged from 20-45 minutes in the CSI group and 39-40 minutes in the attention control group. Within the CSI group, the four habits or routines chosen by the participants to link their medication taking to included: Administration of dog's insulin twice-a-day, driving kids to school, drinking morning coffee prior to work, and drinking power shake after his morning workout. Five of the six randomized participants completed the study. One participant died of causes unrelated to IBD. Demographic differences between the CSI and attention control groups were not calculated due to the samples' low statistical power.

\section{What is the feasibility of the con-} tinuous self-improvement intervention in nonadherent adult inflammatory bowel disease patients?

All participants reported very little (2/5 participants) to just right (3/5 participants) for the amount of time required for participation, with positive comments regarding their experience. Participants wrote they had no significant inconvenience or no disruption with their participation or medication taking. One participant wrote, It took no more time than usual to take medications. A male participant wrote he liked the establishing a good medication taking routine. A female participant wrote, It helped me link my medication taking with another BID task that is easier to remember.

What is the change in the intervention mechanism of personal systems thinking?

Of the four CSI participants, two completed pre/post the Systems Thinking Survey; one participant failed to turn in the initial survey and one participant did not turn in the final survey due to death unrelated to the study. The Systems Thinking Survey mean change in score was $-0.50(\mathrm{SD}=6.36)$, which provided mixed results. One participant's pre-intervention Systems Thinking score was 48 and decreased to 44 at the end of the study, with the other participant score initially was 47 and improved to 52 at the end of the study.

What is the potential effectiveness of the continuous self-improvement intervention in nonadherent adult inflammatory bowel disease patients?

MEMS change scores for the CSI group did increase (MEMS difference mean $=-0.07$; $\mathrm{SD}=0.03$ ) and the MEMS change scores for the attention control group decreased slightly (MEMS difference mean $=0.01, \mathrm{SD}=0.06$ ). However, P-values were not calculated due to the our small sample size and large standard of error, the P-value would not be reliable.

Between the two groups, the change scores were not statistically significant $(\mathrm{P}=0.14$; $\mathrm{CI}$ : $-0.19-0.045)$. Based on the mean change score for the two groups and their corresponding standard deviations, effect size was found to be 1.9. The effect size will assist in estimating the sample size for future study.

\section{Discussion}

The purpose of this pilot study was to assess the feasibility, change in personal systems thinking, and potential effectiveness of a three-month CSI intervention in nonadherent adult IBD patients. Our nonadherence rate of $47.4 \%$ is consistent with the previous IBD literature nonadherence rate of $7-72 \%,{ }^{9}$ and with the chronic disease literature medication nonadherence rate of $50 \% .^{2-4}$

\section{Feasibility}

One purpose of this pilot study was to determine the feasibility of the three-month CSI intervention in adult nonadherent IBD patients. Feasibility of the CSI intervention was found to be positive and without significant participant burden, which was also found in a similar kidney transplant sample. ${ }^{12}$ The single dose of the intervention was adequate to show a trend towards improved adherence without inconveniencing the participants. The goal of any intervention study is to minimize the participant burden while maximizing the dose of the intervention. With a single dose of the CSI intervention in a small sample, trends were noted towards adherence. The participants were active in identifying existing habits and suggesting ways to make their medication taking better.

Table 1. Inflammatory bowel disease categorical demographic data.

\begin{tabular}{lc} 
Variable & $\begin{array}{c}\text { Total sample } \\
(\mathrm{n}=19)\end{array}$ \\
Male & $11(57.9 \%)$ \\
Non-hispanic & $19(100 \%)$ \\
\hline Caucasian & $18(94.7 \%)$ \\
Married & $11(57.9 \%)$ \\
\hline Work full-time & $12(63.2 \%)$ \\
Non-smoker & $18(94.7 \%)$ \\
\hline Some college & $9(47.4 \%)$ \\
Steroid free & $14(73.7 \%)$ \\
\hline Pillbox NOT used & $14(73.7 \%)$ \\
IBD Diagnosis: & $10(52.6 \%)$ \\
Crohn's & $9(47.4 \%)$ \\
\hline Ulcerative colitis &
\end{tabular}

IBD, inflammatory bowel disease.

Table 2. Inflammatory bowel disease continuous demographic data.

\begin{tabular}{lccc} 
Variable & Mean & SD & Range \\
Age (years) & 44.8 & 13.0 & $21.7-67.9$ \\
Age of IBD onset (years) & 32.8 & 12.7 & $18-63$ \\
\hline Number of medications & 5.2 & 4.9 & $1-16$ \\
\hline SD, standard deviation; IBD, inflammatory bowel disease.
\end{tabular}




\section{Personal systems thinking}

The Systems Thinking Survey change scores were mixed. This indicates that the participants receiving the CSI intervention may or may not have shifted their thinking and consequently their behavior towards using personal systems to improve and support medication adherence. However, caution should be used when interpreting these data due to the small sample and lack of psychometric data of the instrument. A larger more diverse sample is needed before conclusions can be made as to the intervention mechanism and whether personal systems thinking improved with the CSI intervention.

\section{Medication adherence}

The change in the medication adherence score for the CSI group was not statistically significant $(\mathrm{P}=0.14)$ though the trends were in the anticipated direction. No statistically significant findings were noted between the CSI and attention control groups, which is similar to other IBD medication adherence intervention studies thus far. ${ }^{23-27}$ Our attention control group showed a weak intervention effect of the IBD education only intervention ( $E S=0.17$ ), consistent with the education alone intervention findings across chronic disease $(\mathrm{ES}=0.29$ $0.61) .28$

Our effect size ( $E S=1.9)$ is larger than the ES calculated across chronic diseases in general (ES: 0.67-1.18). ${ }^{28}$ Attempts were made to calculate effect sizes for the previously reviewed IBD studies; however, not enough data was published to calculate their scores. Our findings are also consistent with a recent systematic review of CSI intervention literature showing improvement in lifestyle and chronic diseases. ${ }^{11}$ A similar study in kidney transplant patients found an equally strong effect size (ES=1.4). ${ }^{12}$ However, our effect size was calculated to estimate sample size for future study and should be considered a crude estimate based on our small sample size.

This IBD medication adherence pilot study is the first to focus on nonadherent participants, utilize electronic monitoring of adherence, and test the innovative CSI intervention in IBD patients. Utilizing a nonadherent, or homogenous, population can increase the study's power, also avoiding the ceiling effect that can be seen in adherent participants. ${ }^{29}$

In the five IBD studies reviewed, this is the first IBD study to utilize electronic monitoring as the adherence measure. Electronic monitoring may be a more expensive monitoring than self-report, but the information gleaned from the data is vital to the CSI intervention (Figures 3 and 4). Electronic monitoring is considered one of the most valid and reliable measures of medication adherence. $16-19,22$

The innovative CSI intervention identifies patients' daily habits that can be linked with medication-taking and help foster change in their personal systems to enhance adherence. One-time delivery of the intervention shows promise, as evidenced by these preliminary data and prior work. ${ }^{12}$ Routine analysis was helpful to assist the participant in identifying potential habits to target for medication taking. With the MEMS feedback, the participant easily identified the change in the system for the days of nonadherence and made inferences for behavior change.

Consistent with prior CSI intervention studies, participants easily accepted the intervention. ${ }^{12}$ The ability to deliver the intervention in the clinical setting by the PI with patient acceptance indicates that the intervention may more broadly translate into the clinical setting.

The change in personal systems thinking was mixed, but the sample size was small and the instrument lacks psychometric data. Personal system thinking is the concept CSI is designed to change; with no psychometric data to validate the instrument, the tool may not measure what it is intended to measure which threatens the internal validity of the study. Further testing of the instrument should strengthen the accuracy of the tool.

The World Health Organization (WHO) recommends that patients need to be supported and not blamed for their nonadherence. The WHO also suggests that integrating medication taking with a daily habit may improve adherence. ${ }^{1}$ Russell, Ruppar, and Matteson (2011) recommend shifting attention to personal systems change interventions, shaping routines through the PDCA process, and selfmonitoring as a means of enhancing medication adherence behaviors. ${ }^{30} \mathrm{~A}$ systematic review found behavioral interventions with self-monitoring and feedback were effective. ${ }^{5}$ This pilot study contributes further evidence that behavioral system based personal level interventions have potential to shape patient's behaviors.

Limitations of the study include the small sample size, a non-equivalent control group, short dose and duration of the CSI intervention, attrition bias due to the loss of three nonadherent participants, possible testing bias as the system survey was repeated, and the survey possesses no psychometric data. In addition, generalizability of the findings is limited to those taking once and twice daily dosed medications. These limitations are fewer than the previous IBD adherence studies as these studies were not fully powered, had small sample sizes, utilized self-report instruments, lacked specific operational definitions of medication adherence, possessed short duration and dose of intervention, and lacked a theorybased intervention (in three of the five studies). Generalizability of the study findings is limited due to the small sample size and the homogenous sample.

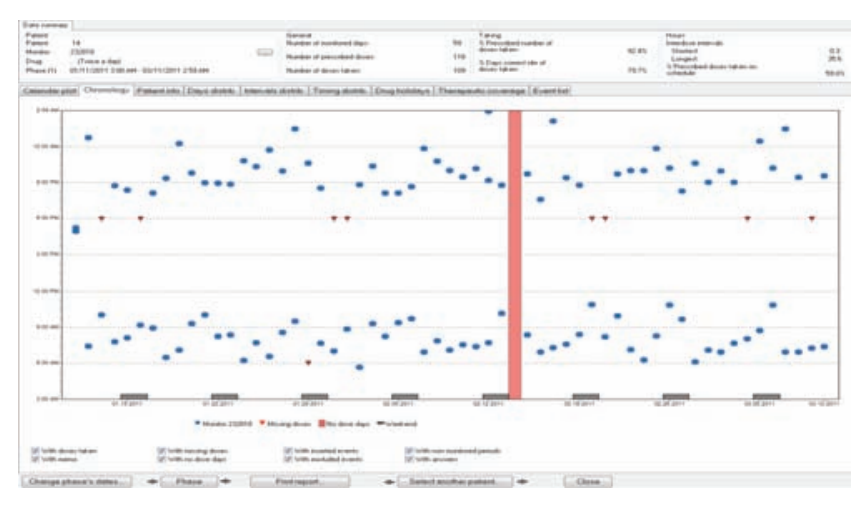

Figure 3. Medication Event Monitoring System report before continuous self-improvement.

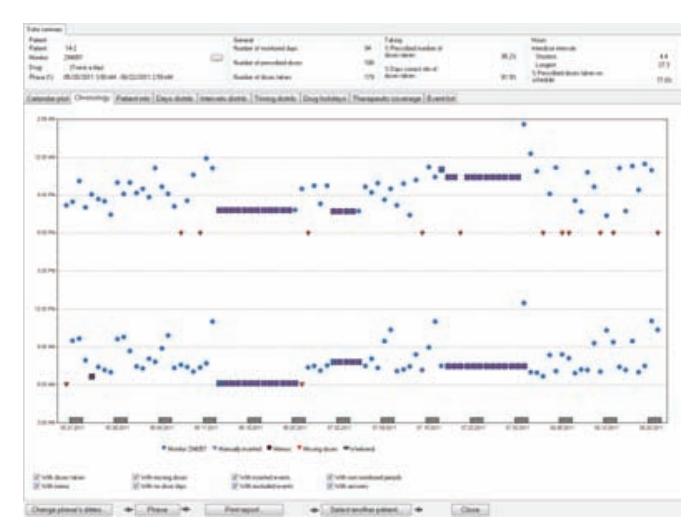

Figure 4. Medication Event Monitoring System report after continuous self-improvement. 


\section{Conclusions}

In conclusion, this pilot study examined the feasibility, intervention mechanism, and potential effectiveness of CSI to enhance maintenance medication adherence in nonadherent adults IBD patients. Within the context of the current evidence, this study contributes to the growing medication adherence literature within IBD. In contrast to the existing IBD studies using cognitive-based interventions, this pilot study with a small nonadherent sample, used an innovative intervention based on personal systems theory using electronic monitoring. A fully powered study should further examine the effectiveness of the CSI intervention in IBD patients.

\section{References}

1. Sabaté, E. Adherence to long-term therapies: evidence for action. Geneva: World Health Organization, 2003.

2. Roter DL, Hall JA, Merisca R, et al. Effectiveness of interventions to improve patient compliance: a meta-analysis. Med Care 1998;36:1138-61.

3. Peterson AM, Takiya L, Finley R. Metaanalysis of trials of interventions to improve medication adherence. Am J Health Syst Pharm 2003;60:657-65.

4. McDonald HP, Garg A, Haynes RB. Interventions to enhance patient adherence to medication prescriptions: scientific review. JAMA 2002;288:2868-79.

5. Kripalani S, Yao X, Haynes RB. Interventions to enhance medication adherence in chronic medical conditions: a systematic review. Arch Intern Med 2007;167:540-50.

6. Loftus EV Jr. Clinical epidemiology of inflammatory bowel disease: incidence, prevalence, and environmental influences. Gastroenterology 2004;126:1504-17.

7. Lichtenstein GR. Current research in Crohn's disease and ulcerative colitis: highlights from the 2010 ACG Meeting. Gastroenterol Hepatol 2010;6:3-14.

8. Hanauer SB. Review article: high-dose aminosalicylates to induce and maintain remissions in ulcerative colitis. Aliment Pharmacol Ther 2006;24:37-40.

9. Jackson CA, Clatworthy J, Robinson A, Horne R. Factors associated with nonadherence to oral medication for inflammatory bowel disease: a systematic review. Am J Gastroenterol 2010:105:525-39.

10. Hawthorne AB, Rubin G, Ghosh S. Review article: medication non-adherence in ulcerative colitis--strategies to improve adherence with mesalazine and other maintenance therapies. Aliment Pharmacol Ther 2008;27:1157-66.

11. Matteson, M.L., Russell, C.L. Systematic review of continuous self-improvement interventions. 2011. [In press].

12. Russell CL. A clinical nurse specialist-led intervention to enhance medication adherence using the plan-do-check-act cycle for continuous self-improvement. Clin Nurse Spec 2010;24:69-75.

13. Gustafson DH, Cats-Baril WL, Alemi F. Systems to support health policy analysis: theory, models, and uses. Ann Arbor: Health Administration Press; 1992.

14. Alemi F, Neuhauser D, Ardito S, et al. Continuous self-improvement: systems thinking in a personal context. Jt Comm J Qual Improv 2000;26:74-86.

15. Alemi F, Neuhauser D, eds. A thinking person's weight loss and exercise program. Fairfax: George Mason University; 2006.

16. Russell CL, Conn V, Ashbaugh C, et al. Intra-subject medication adherence patterns. Clin Nurs Res 2007;16:153.

17. Denhaerynck K, Schaefer-Keller P, Young $\mathrm{J}$, et al. Examining assumptions regarding valid electronic monitoring of medication therapy: development of a validation framework and its application on a European sample of kidney transplant patients. BMC Med Res Methodol 2008;8:5.

18. De Geest S, Vanhaecke J. Methodological issues in transplant compliance research. Transplant Proc 1999;31:81S-3.

19. Reikert KA. Integrating regimen adherence assessment into clinical practice. In: 0'Donohue WT, Levensky ER (Ed.). Promoting treatment adherence: a practical handbook for health care providers. Thousand Oaks: Sage Publishers; 2006.

20. De Geest S, Schafer-Keller P, Denhaerynck $\mathrm{K}$, et al. Supporting medication adherence in renal transplantation (SMART): a pilot RCT to improve adherence to immunosuppressive regimens. Clin Transplant 2006;20:359-68.

21. Kruse W, Weber E. Dynamics of drug regimen compliance-its assessment by microprocessor-based monitoring. Eur J Clin Pharm 1990;38:561-5.

22. Russell CL, Conn VS, Ashbaugh C, et al. Medication adherence patterns in adult renal transplant recipients. Res Nurs Health 2006;29:521-32.

23. Waters BM, Jensen L, Fedorak RN. Effects of formal education for patients with inflammatory bowel disease: a randomized controlled trial. Can J Gastroenterol 2005;19:235-44.

24. Moss AC, Chaudhary N, Tukey M, et al. Impact of a patient-support program on mesalamine adherence in patients with ulcerative colitis--a prospective study. J Crohns Colitis 2010;4:171-5.

25. Cook PF, Emiliozzi S, El-Hajj D, McCabe MM. Telephone nurse counseling for medication adherence in ulcerative colitis: a preliminary study. Patient Educ Couns 2010;81:182-6.

26. Moshkovska T, Stone M, Baker R., et al. Qualitative investigation of patient adherence to 5-aminosalicylic acid therapy in patients with ulcerative colitis. Inflamm Bowel Dis 2008;14:763-8.

27. Keefer L, Doerfler B, Artz C. Optimizing management of Crohn's disease within a project management framework: results of a pilot study. Inflamm Bowel Dis 2012;18:254-60.

28. Conn VS, Hafdahl AR, Cooper PS, et al. Interventions to improve medication adherence among older adults: metaanalysis of adherence outcomes among randomized controlled trials. Gerontologist 2009;49:447-62.

29. Polit D, Beck, C. nursing research: principles and methods. 9th Ed. Philadelphia: Lippincott, Williams \& Wilkins; 2012.

30. Russell CR, Ruppar TR, Matteson ML. Improving medication adherence: moving from intention and motivation to a personal systems approach. Nurs Clin North Am September 2011;46:271-81. 